Revista de Comunicación y Salud, 2019, Vol. 9, n 1, pp. 65-76

Editado por Cátedra de Comunicación y Salud

ISSN: 2173-1675

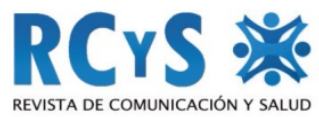

Enviado $12 / 10 / 2018$

Aprobado 18/11/2018

\title{
EDUCACIÓN EN SALUD SEXUAL, UNA NECESIDAD QUE VA MÁS ALLÁ DEL APORTE DE INFORMACIÓN
}

\author{
Sexual Health Education, a need that goes beyond the provision of information
}

\author{
Albino José Rojas Piñango ${ }^{1}$ \\ Universidad Complutense de Madrid
}

\begin{abstract}
Resumen
El presente ensayo surge de una revisión documental del problema creado por el elevado índice de Embarazo en adolescentes en Latino América y las diferentes iniciativas que se han producido para tratar de minimizarlo. Muchas se apoyan en enfoques biologicistas que refuerzan lo que se denomina genitalidad; según la cual, la sexualidad se asocia con la reproducción, con la participación casi exclusiva de los órganos genitales. Parte de un programa denominado: Metas Educativas 2021: la educación que queremos para la generación de los Bicentenarios, el cual constituye una de las iniciativas de mayor envergadura y significación que se ha puesto en marcha en los últimos años. Debe dar una idea de las actitudes, de las presiones, conciencia de las alternativas y sus consecuencias; aumentar el amor, el conocimiento propio, mejorar la toma de decisiones y la técnica de la comunicación. Cuando se hace referencia a la Educación en Sexualidad se ha de tener una visión completa de la complejidad del ser sexuado, y partir de que la sexualidad es una parte integral de la personalidad de todo ser humano. De allí que debe ser abordada desde la integralidad del ser. Destaca que la solución está en abordarla desde las dimensiones afectivas y espirituales superando los esquemas biologicistas de salud y riesgo.
\end{abstract}

Palabras clave: salud, salud sexual, educación en sexualidad, afectividad, espiritualidad.

\section{Abstract}

This essay arises from a documentary review of the problem created by the high rate of pregnancy in adolescents in Latin America and the different initiatives that have been produced to try to minimize it. Many rely on biologicist approaches that reinforce what is called genitality; according to which, sexuality is associated with reproduction, with the almost exclusive participation of the genital organs. Part of a program called: Educational Goals 2021: the education we want for the generation of the Bicentennial, which is one of the initiatives of greater scope and significance that has been launched in recent years. It should give an idea of attitudes, of pressures, awareness of alternatives and their consequences; increase love, self-knowledge,

\footnotetext{
${ }^{1}$ Autor para correspondencia: Albino José Rojas Piñango arojas_5@hotmail.com
} 
improve decision-making and the technique of communication. When referring to Education in Sexuality, we must have a complete vision of the complexity of being sexed, and that sexuality is an integral part of the personality of every human being. Hence, it must be approached from the integrality of being. It emphasizes that the solution is to approach it from the affective and spiritual dimensions overcoming the biologicist schemes of health and risk.

Keywords: health, sexual health, education in sexuality, affectivity, spirituality.

\section{Cómo citar el artículo}

Rojas Piñango, A. J. (2019). Educación en Salud Sexual, una necesidad que va más allá del aporte de información. Revista de Comunicación y Salud, 9(1), pp. 65-76. doi: http://doi.org/10.35669/revistadecomunicacionysalud.2019.9(1).65-76

La Organización de Estados Iberoamericanos (OEI, 2012) promovió a partir de un análisis consensuado sobre la problemática latinoamericana en el campo un programa denominado: Metas Educativas 2021: la educación que queremos para la generación de los Bicentenarios, el cual constituye una iniciativa importante y significativa. En la opinión de su Secretario General, Álvaro Marchesi Ullastres, el logro de sus objetivos contribuirá de forma decisiva al desarrollo de los pueblos y al bienestar de los ciudadanos. Su finalidad es ambiciosa y se orienta a mejorar la calidad y la equidad en la educación. Para lograrlo se requiere con apremio unir esfuerzos conjuntos que permitan orientar las políticas de cada estado hacia metas que potencien la educación en valores para una ciudadanía democrática y activa, que se oriente hacia la cultura de la paz, la valoración de la democracia, el respeto al ambiente y la igualdad de género, la promoción de la salud para alcanzar una mejora significativa en la calidad de vida de la población. Sin embargo, ella no puede por sí sola resolver los problemas que la sociedad ha generado, sino que exige para ello que se produzcan cambios significativos en otros ámbitos de la sociedad.

El Fondo de Poblaciones de las Naciones Unidas UNFPA alerta para la región latinoamericana incrementos significativos en violencia, homicidio, suicidios, embarazos en adolescentes, consumo de alcohol y estupefacientes desde la adolescencia. Este programa se constituye en un eje de referencia que desafía a propiciar una base sólida que facilite que el sistema educativo iberoamericano se enfrente con mayores garantías de éxito a uno de los desafíos que tiene planteado, como lo es el lograr que todos los alumnos accedan a una educación de calidad; garantizando el cumplimiento de ciertas condiciones imprescindibles para el aprendizaje, como la salud y la alimentación de la población que le corresponde atender y lo preparen para el desarrollo integral de su personalidad.

El mismo programa hace énfasis en lo social y participativo y será objeto de una constante evaluación y seguimiento por parte de los responsables de las políticas educativas en cada nación iberoamericana. Estos pilares fundamentales consiguen su correspondencia directa en las once metas generales que plantearon. De las cuales merecen especial mención, la primera y la quinta que apuntan a:

1. Reforzar y ampliar la participación de la sociedad en la acción educadora. 
2. Ofrecer un currículo significativo que asegure la adquisición de las competencias básicas para el desarrollo personal y el ejercicio de la ciudadanía democrática (Organización de Estados Iberoamericanos, 2012).

Laak (1996), analiza las cinco grandes dimensiones de la personalidad; también llamadas rasgos o factores que constituyen los pilares básicos que captan la esencia de las diferencias individuales. Se desarrollan en base a influencias genéticas como ambientales. Estudios realizados han mostrado que se trata de dimensiones universales y pueden utilizarse para describir la personalidad de individuos de culturas muy diferentes. Estas dimensiones son:

I. Extroversión (Surgencia): afecto, gregarismo, asertividad, actividad, búsqueda de emociones, emociones positivas.

II. Agradabilidad (Simpatía): confianza, honradez, altruismo, cumplimiento, modestia, sensibilidad.

III. Consciencia (Conformidad, Seguridad): competencia, orden, obediencia, lucha por el logro, autodisciplina, reflexión.

IV. Neuroticismo (Estabilidad Emocional, Inquietud): Ansiedad, hostilidad colérica, depresión, timidez, impulsividad, vulnerabilidad.

V. Apertura a la Experiencia (Cultura, Intelecto, "Intelectancia"): fantasía, estética, sentimientos, acciones, ideas, valores.

Las metas señaladas anteriormente podrían conjugarse para promover el desarrollo integral de la personalidad orientada hacia la participación social en la construcción de un currículo significativo para el desarrollo y abordaje de uno de los aspectos cruciales de la personalidad del ser como lo es la sexualidad. Este aspecto es abordado por la Educación en Sexualidad de una manera que, según el Fondo de Poblaciones de las Naciones Unidas (UNFPA, 2011) y la Organización Mundial de la Salud (OMS), no ha sido efectiva según los indicadores de embarazo en adolescentes, infecciones de VIH - Sida, violencia doméstica y desigualdad de géneros que se presentan en los países de Latinoamérica.

Este enfoque representa lo que ha constituido el enfoque de Salud Sexual Reproductiva. En función de lo que el UNFPA promueve, desde el año 2003, el Programa de Educación en Sexualidad Humana y Salud Sexual Reproductiva mediante un convenio con la Universidad Pedagógica Experimental Libertador (UPEL) para integrar formalmente la Educación Integral de la Sexualidad (EIS) en el currículo que ofrece a sus estudiantes. Como resultado de ese trabajo intersectorial, en el que participaron especialistas de los ocho institutos que conforman la UPEL, en febrero de 2012 se presentaron las Líneas Estratégicas Curriculares para la Educación de la Sexualidad en el Subsistema de Educación Básica. Este nuevo currículo contempla la promoción de estilos de vida saludables y la prevención de problemas sociales y de salud en niños, niñas y adolescentes.

Por su parte la OMS y La Organización Panamericana de Salud (OPS), junto con el Ministerio de Educación (1989), diseñaron un programa de capacitación permanente para los docentes de Educación para la Salud, formándose una comisión Regional encargada de elaborar estrategias para actualizar al docente en Educación para la Salud y la promoción de la salud sexual y reproductiva de los educandos del país. 
Estos esfuerzos que se mencionan surgen como una respuesta a lo que consideran el bajo nivel de logro que ha tenido la Educación en Sexualidad en Venezuela y muchos países de Latinoamérica; según los indicadores de embarazo en adolescentes, infecciones de VIH - Sida, violencia doméstica y desigualdad de géneros. Unido a otros indicadores socio económico como el aumento del índice de mujeres como jefe de familia, violencia doméstica y divorcio, que dan cuenta de ello.

La Fundación Escuela de Gerencia Social (2006), con apoyo de la representante del UNFPA - Venezuela Luz Beltrán Molina, promovió una investigación sobre el embarazo en adolescente. Allí plantearon algunos indicadores que describen la situación como un problema de salud pública en Venezuela; estos son:

1. Los principales problemas de salud sexual y reproductiva son la elevada cifra de mortalidad materna por causas prevenibles y el embarazo en adolescentes.

2. Las cifras disponibles que perfilan la situación de la maternidad temprana son: EI $50 \%$ de las adolescentes venezolanas se inicia sexualmente antes de los 19 años y el $10 \%$ antes de los 15 . En zonas menos urbanizadas y rurales la edad disminuye, el $30 \%$ se inicia antes de los 15 años y el $70 \%$ antes de los 19 .

3. Las adolescentes no suelen usar métodos anticonceptivos en sus primeras relaciones sexuales. A pesar de que 9 de cada 10 adolescentes conoce los métodos anticonceptivos, sólo 1 de cada 10 los usa.

4. Solamente 1 de cada 5 adolescentes conoce su período fértil, lo que torna en muy riesgoso el uso del método del coito interrumpido.

5. La tasa de fecundidad de las jóvenes de 15 a 19 años se estima en $97 \%$, lo que quiere decir que 97 jóvenes por cada mil ha tenido un hijo.

6. El $21 \%$ de los nacimientos vivos registrados en el país ocurre en adolescentes entre los 15 y 19 años.

7. El embarazo interrumpe los estudios: 2 de cada 5 adolescentes dejan la escuela como consecuencia del embarazo y a mayor educación, menos embarazos. Las adolescentes más pobres y menos instruidas tienen 4 veces más riesgo de embarazarse que las menos pobres e instruidas.

8. El $6.4 \%$ de las adolescentes había tenido un aborto.

9. La tercera causa de muerte en las mujeres de 15-19 años obedece a complicaciones obstétricas.

De acuerdo a lo anterior estos indicadores de índole socioeconómico, educativo y cultural perfilan la realidad que se presenta en Venezuela y que la ubican entre los países de Latinoamérica con las más elevadas cifras de embarazos tempranos. Según la OMS/OPS se refiere a aquellos que se presentan en edades menores de 19 años. Proyectando esta situación al ámbito internacional el Fondo de Naciones Unidas para la Infancia (UNICEF, 2007) señala que:

El $42 \%$ de las adolescentes en edades de 14 a 19 años, en todo el mundo, están embarazadas, el 33,95\% ya son madres, dos de cada cinco de ellas, no habían salido de la primaria. Asimismo, las niñas menores de 15 años tienen cinco veces más probabilidad de morir en el embarazo que las mujeres mayores de 20 años, constituyendo esta situación un problema de salud pública... (p.2)

Esto denota que existe una realidad que supone atención, y es que se la asocia a lo que se conoce como Salud Sexual Reproductiva. Reforzando la idea de que la Sexualidad se asocia principalmente a la reproducción, cuando en realidad va mucho más allá. De allí que cuando se le plantea a las personas con qué asocian el

Revista de Comunicación y Salud, 2019, Vol. 9, nº 1, pp. 65-76 
termino de Sexualidad un elevado porcentaje señala que se relaciona con la práctica de relaciones sexuales. $Y$ es precisamente esa práctica, que se realiza sin protección ni prevención, la que ha generado la situación descrita.

Con relación a la visión que tiene el imaginario colectivo de lo que se refiere a la sexualidad generalmente se aproxima a la genitalidad y por ende a la reproducción. Por ejemplo, en el caso de la promoción del uso del preservativo masculino las principales creencias para no usarlo son que, afectarían la sensación de placer, representan falta de confianza hacia la pareja y, en caso de los varones, no es responsabilidad suya, estos dos últimos motivos reflejan la fuerte influencia de los estereotipos de género. Otras causas, son el miedo a su rotura, la dificultad para obtenerlos y la vergüenza al comprarlos.

Desde esta visión, la problemática que se presenta no obedece a la falta de información relacionada con las relaciones sexuales y el embarazo, como ocurría en las generaciones anteriores. En el país se vincula el embarazo en la adolescencia con la cultura, con el proyecto en el ciclo de vida, con la pobreza y la educación. En este sentido, en la educación formal, específicamente, en el currículo de la Educación Primaria y Secundaria se han incluido contenidos que, de alguna forma promueven el ejercicio de una sexualidad sana y responsable. En las instituciones educativas muchos docentes han recibido charlas y talleres de actualización para el abordaje del tema con sus estudiantes. Inclusive en la red se puede conseguir, orientado por los padres y maestros, información actualizada para los niños, niñas, adolescentes y adultos.

Sin embargo, estos elementos como la cultura, el proyecto en el ciclo de vida, la pobreza y la educación son más determinantes en el establecimiento de ciertas prácticas que generan conductas de riesgos responsables de los indicadores precitados. En muchos casos el machismo, la sumisión de la mujer, el estereotipo del mago, el cazador y el superhéroe promueven estas conductas que conllevan las consecuencias previamente establecidas. $Y$ eso se asume como normal y repercute en el rol de los padres que, lejos de reorientar esas conductas las promueven, con la idea fija de que así "debe ser". De igual forma el docente que también es formado bajo estas condiciones obedece a ellas y las promueve en sus estudiantes.

Afortunadamente han surgido iniciativas que, de una forma u otra buscan dar respuestas asertivas a esta situación. El Organismo Regional Andino de Salud en el Convenio Hipólito Unanue (ORAS-CONHU, 2008), presenta el Plan Andino para la Prevención del Embarazo Adolescente (PLANEA) que constituye un punto de encuentro de representantes de alto nivel y equipos técnicos de los sectores de salud, protección social, juventud, educación y bienestar social de Bolivia, Colombia, Chile, Ecuador, Perú y Venezuela y otras organizaciones regionales como la Organización Iberoamericana de la Juventud (OIJ) y Family Care International (FCl).

Allí se planteó que para abordar la prevención y la atención al embarazo temprano deben realizarse iniciativas multisectoriales y multidisciplinares desde los mismos gobiernos, que cuente con la participación activa de los propios adolescentes y jóvenes a quienes se dirigen. La prevención de estos embarazos implica abordar los factores de riesgo que lo determinan y la promoción del 
desarrollo humano, impulsando la autonomía haciendo énfasis en el proyecto de vida de los jóvenes y su seguimiento.

Podría decirse que en las políticas públicas que se diseñen orientadas a garantizar la salud sexual y reproductiva debe hacerse hincapié en la prevención y la atención al embarazo temprano en el marco de los derechos humanos como un eje central de forma integral, además de constituirse en componentes prioritarios en las políticas sectoriales de salud y educación. De allí que se necesitan políticas a largo plazo y amplio alcance que contrarresten los efectos de la pobreza y la falta de oportunidades para el desarrollo pleno de los jóvenes y adolescentes, sin diferenciación de género. Promoviendo en todo momento el desarrollo humano en sus facultades, la autonomía y su proyecto de vida.

Uno de los avances alcanzados en el campo de las políticas públicas educativas en esa época lo ostenta el Ministerio del Poder Popular para la Educación (MPPE, s/f) cuando, entre los años 2.000 y 2.005 presenta una propuesta pedagógica que aspiraba promover la formación permanente de los corresponsables de la educación, así como la elaboración de recursos para el aprendizaje, partiendo de la investigación, la creatividad y la innovación, dando cumplimiento a lo establecido en la normativa legal vigente para ese momento. Esto representó un aporte valioso en el enfoque educativo que se le dio a la Educación en Sexualidad. El mismo trascendió lo informativo - preventivo y se iniciaba desde el preescolar.

Desafortunadamente no se le dio continuidad y allí quedo una iniciativa que habría dado significativos avances en la promoción del desarrollo y formación integral de los ciudadanos; ya que buscaba promover la toma de decisiones responsables, la práctica de estilos de vida saludables, así como conocimientos, actitudes, valores y comportamientos positivos hacia la vivencia de una sexualidad placentera y responsable, mediante la articulación de la familia, la escuela y la comunidad. Para ese momento el MPPE (ob.cit.) asumió, y así parecía serlo, el compromiso de que con esta política educativa se estaría garantizando; "la implementación de la educación de la sexualidad con un enfoque democrático, participativo, corresponsable y protagónico en el marco de los derecho humanos, que conlleve a estrategias de trabajo inter y transdisciplinarias" (p. 37).

Dentro de los desafíos que se plantearon estaba la necesidad de una mayor participación por parte de la familia en las actividades formativas que se realizaban en los planteles educativos. No solo era el hecho de que fueran informados o invitados a charlas o talleres en las escuelas, sino que las familias pudieran sentir que podían contar con la escuela y el liceo para que los apoyara en el fortalecimiento de espacios e iniciativas orientadas a favorecer una buena comunicación familiar en el tema.

Esto es importante ya que, la participación de la familia y la comunidad en las actividades que se desarrollan desde las escuelas en la actualidad es muy escasa. Existe una separación importante en los intereses que orientan a cada uno de estos sectores. La situación país que se vive, según refleja la Encuesta sobre Condiciones de Vida en Venezuela (ENCOVI 2017) muestra que el empeoramiento de la calidad de vida, no sólo se relaciona con la pérdida del poder de compra, sino que afecta las dimensiones más estructurales de los hogares, ha sido determinante en que esto 
ocurra. Esto conlleva a la necesidad de fomentar y dar las facilidades para la formación continua de las y los profesores del sistema, de manera que incorporen en sus prácticas pedagógicas los contenidos, habilidades, metodologías y actitudes necesarias para el desarrollo pleno e integral de la sexualidad de sus alumnos y alumnas sugeridas por la misma sociedad en la que ejercerán su acción docente; incorporando la dimensión humana de la afectividad.

Considerando posibles soluciones a la situación descrita, en los países latinoamericanos y, en el caso especial de Venezuela, se hace urgente la propuesta de implementar acciones que den respuesta inmediata a la situación que en materia de Educación en Sexualidad reclama la sociedad. Esto pasa por profundizar en la promoción de una sexualidad integral, placentera y responsable, lo cual tendrá resonancia en contextos sociales, económicos e impactará positivamente en el desarrollo del país, Para lograrla es útil reivindicar los alcances y dimensiones de la Sexualidad en los términos en que lo expuso la Organización Mundial para la Salud (1983):

La educación sexual debe abarcar mucho más que la información. Debe dar una idea de las actitudes, de las presiones, conciencia de las alternativas y sus consecuencias. Debe de aumentar el amor, el conocimiento propio, debe mejorar la toma de decisiones y la técnica de la comunicación (p. s/n).

Una de las acciones ejecutadas fue en 2009 cuando en Ciudad de México se realizó la reunión de Ministros de Salud y Educación para Detener el VIH y cualquier otra ITS en Latinoamérica y el Caribe, en el marco de la XVII Conferencia Internacional de SIDA, con el objetivo de fortalecer la respuesta a la epidemia del $\mathrm{VIH}$ en el contexto educativo formal y no formal. Entre los aspectos que consideraron fue que un porcentaje considerable de las personas jóvenes inicia su actividad sexual a edades tempranas y en la mayoría de estos encuentros sexuales no se utiliza protección para prevenir infecciones sexualmente transmisibles.

Los representantes de los Ministerios de Salud de los países asistentes a esta reunión coincidieron en considerar que la educación integral en sexualidad desde la infancia, favorece la adquisición gradual de la información y de los conocimientos necesarios para el desarrollo de las habilidades y de las actitudes apropiadas para vivir una vida plena y saludable, con lo cual se podrían reducir riesgos en materia de salud y reproducción. La misma debía incorporar de manera articulada, equilibrada y consensuada con audiencia y comunidad los aspectos: éticos, biológicos, emocionales, sociales, culturales y de género, así como temas referentes a la diversidad de orientaciones e identidades sexuales conforme al marco legal de cada país, para así generar el respeto a las diferencias, el rechazo a toda forma de discriminación y para promover la toma de decisiones responsables e informadas con relación al ejercicio de su sexualidad; ello se reflejaría en su autoestima, respeto y relaciones de género positivas desde la infancia que lo podría llevar a postergar el inicio de sus relaciones sexuales, hasta que se alcance la madurez psicológica para hacerlo. Esta posición está en congruencia con la posición del PNUD, capítulo Venezuela y la línea de investigación de la Universidad Pedagógica Experimental Libertador (UPEL) que promueve una Educación en Sexualidad que dé respuestas oportunas e integrales a la situación que se vive en los países latinoamericanos. 
En la mencionada Cumbre Ministerial se proyectó que para el año 2015, se habría reducido en $75 \%$ la brecha en el número de escuelas que para ese momento no habían institucionalizado la Educación Integral en Sexualidad en los centros educativos bajo la jurisdicción de los Ministerios de Educación. Así mismo, se esperaba la reducción en $50 \%$ de la brecha en adolescentes y jóvenes que carecían de cobertura de servicios de salud para atender apropiadamente sus necesidades de salud y reproducción. Convencidos de que estas acciones reflejan el compromiso de los países con las niñas y los niños, adolescentes y jóvenes de América Latina y el Caribe, con los países hermanos, y con la comunidad mundial.

En el año 2012 se hizo una evaluación de los logros alcanzados en cada país que suscribió esta Declaración Ministerial, y se evidenció que en general se ha avanzado en un $58 \%$. Los porcentajes de avance particular son los siguientes: Cuba, 99; Ecuador, 86; Argentina, 82; Costa Rica, 80; Colombia, 79; Uruguay, 71; Brasil, 69; México, 68; Guatemala, 67; Nicaragua, 66; El Salvador, 60; Honduras y Perú, 51;Panamá, 40; Chile, 35; Venezuela, 34; Paraguay y República Dominicana, 30 y Bolivia, 24.

Especificamente en la República Bolivariana de Venezuela en el Plan de la Patria (2013-2019), oficialmente llamado el Segundo Plan Socialista de Desarrollo Económico y Social 2013-2019, inicialmente en junio de 2012 y posteriormente en diciembre, de ese mismo año. El documento está basado en la ideología de Hugo Chávez (chavismo) y en el socialismo del siglo XXI. En lo relacionado a la sexualidad en la sección 2, numerales del 2.2.4.1, 2.2.4.2 y 2.2.4.3 plantea: Consolidar la equidad de género con valores socialistas, garantizando y respetando los derechos de todos y todas, y la diversidad social. Profundizar la participación política y protagónica de las mujeres; Incorporar la perspectiva de la igualdad de género en las políticas públicas promoviendo la no discriminación y la protección de los grupos socialmente vulnerables y generar políticas formativas sobre la perspectiva de igualdad de género y de diversidad sexual. Sin embargo hasta la fecha nada se ha cumplido y menos se han generado las Políticas Públicas que pudieran servirle de soporte para lograr esas metas.

Lo anterior es reflejo de ese $34 \%$ de avance que se le asigna al país, y según los últimos datos oficiales disponibles del 2014, aportados por UNICEF, Venezuela tiene una de las tasas de fecundidad adolescente más altas de Suramérica, en donde cada año nacen 93 bebés por cada 1000 adolescentes entre 15 y 19 años, lo que se traduce en que del total de nacimientos en el país, $23 \%$ son de madres adolescentes y según La Prensa Lara (2018, documento en línea) el país es el tercero con más embarazos adolescentes, detrás de Ecuador y Honduras.

Esto denota que en el país la situación sigue siendo delicada y poco se ha avanzado desde 2008. Cuando se hace referencia a la Educación en Sexualidad se ha de tener una visión completa de la complejidad del ser sexuado, y partir de la consideración de que la sexualidad es una parte integral de la personalidad de todo ser humano. Asimismo, hay que tener presente que ésta se construye a través de la interacción entre el individuo y las estructuras sociales, y que su desarrollo pleno es esencial para el bienestar individual, interpersonal y social.

En materia del abordaje de la sexualidad y la promoción de su educación a nivel formal e informal, a lo largo de la historia reciente se han puesto de manifiesto 
diferentes modelos que conviven en la actualidad, entremezclándose y distorsionando mensajes. Resulta evidente que deben existir directrices o pilares fundamentales que tipifiquen la realidad de una determinada sociedad en esta materia, pero, no deben dejar de considerarse los matices propios de la localidad y cotidianidad de la comunidad beneficiaria de tales políticas. Aquí juegan un papel importante las representaciones sociales responsables del establecimiento del imaginario social, que al final delimitarán la cosmovisión de la comunidad y su comportamiento al respecto.

Hay que partir de lo expresado por Weeks (1998), cuando dice que la sexualidad: ...es un concepto profundamente problemático, y no hay respuestas fáciles a los desafíos que plantea. Pero si empezamos por hacer las preguntas correctas, entonces tal vez podremos encontrar el camino a través del laberinto. Al final del viaje no hallaremos una prescripción de conducta correcta, pero tal vez encontremos un marco que nos permita aceptar la diversidad, y volvamos a encontrar, en la sexualidad, nuevas oportunidades para las relaciones creativas, la acción y la elección (p. 22).

Para este autor la sexualidad no ha sido formulada como una manera de estudiarla, de entenderla, de hacer sentido académico. Las personas tienen experiencias ligadas a su sexualidad, las han vivido desde toda la vida. Pero la manera de hablar de ella es relativamente nueva. Más o menos desde la Revolución Industrial es que se hace. La simple idea de ella prácticamente es imposible traducirlo en las culturas asiáticas (China o Japón, por ejemplo). Porque la cultura oriental no tiene el desarrollo conceptual que se tiene en occidente. Cuando se está frente a una abstracción, todo depende cómo se haga esa abstracción para saber de qué es de lo que se está hablando.

Si bien las distintas culturas interpretan de forma variable la sexualidad, los occidentales han sido regidos por la tradición judeo - cristiana que ha tendido a ver en ella una fuente de aprensión y conflicto moral. Esta doctrina juega un papel fundamental en la definición de la subjetividad de la persona, de la moralidad, del pecado, de la normalidad y de la anormalidad. Esto se evidencia en el dualismo filosófico establecido entre el espíritu y la carne, entre la mente y el cuerpo. En la cultura occidental, el cuerpo se repudia como la parte indigna, impura o pecaminosa; el espíritu es la parte noble que conecta la existencia del ser a lo divino.

Weeks (ob.cit.), generaliza sus planteamientos puntualizando que la sexualidad es un universo complejo en el cual intervienen aspectos tanto biológicos, como psicológicos y sociales. Engloba una serie de condiciones culturales, sociales, anatómicas, fisiológicas, emocionales, afectivas y de conducta, relacionadas con el sexo que caracterizan de manera decisiva al ser humano en todas las fases de su desarrollo.

Por otra parte desde una perspectiva sociobiológica, Reiss (1967), propuso entender la sexualidad como "el conjunto de guiones culturales compartidos acerca de las conductas eróticas que se supone inducen a la excitación erótica y a las respuestas genitales" (p. 45). Tales conductas tienen dos consecuencias universales: están revestidas de importancia cultural y llevan a la formación de vínculos entre los participantes. 
Los planteamientos de estos autores contextualizan la realidad que se vive y la que se aspira establecer, junto con otros que serán abordados más adelante, permiten asumir que la sexualidad en su esencia está desligada de los aspectos Salud y Reproducción. Tradicionalmente se habla de Salud Sexual - Reproductiva para abordar el estudio de la Sexualidad y más específicamente de la Educación en Sexualidad. No obstante la sexualidad por si sola representa un constructo teórico que va más allá de ambas dimensiones. Ella de por si toca la esencia del ser humano, como persona que siente, piensa y actúa. Forma parte de su personalidad. Es por ello que a partir de este punto del estudio se hablará solo de sexualidad como un proceso signado por la complejidad, en el contexto planteado por Morín (2004), en el que además de las cuestiones relacionadas con la excitación sexual, hay elementos diferentes, como la identidad o la afectividad que la cercanía erótico sexual produce con mucha frecuencia que no pueden ser ignorados al elaborar un concepto formal de sexualidad.

Esta complejidad de la sexualidad hace que los seres humanos se diferencien del resto de los seres vivos. Ellos poseen necesidades que van más allá de las naturales, y esta característica es la que les da su verdadera especificidad. Lo que para los animales es relativamente simple, en el humano se vuelve complejo.

Es así como la sexualidad puede ser vista como un elemento muy importante de la vida humana y hasta podría decirse que modula la percepción que el individuo tiene de sí mismo y del mundo del cual forma parte desde la visión de su sexo género. De allí que, para entenderla es necesario, por lo tanto, contextualizarla en un complejo de relaciones que la ubiquen en su auténtica dimensión.

Las necesidades sexuales para el ser humano no son, como en el resto de los seres vivos, un llamado a la reproducción, sino que se relacionan con la autoestima, con el placer, con los sentimientos, con la moral, con las costumbres, con la religión, con el derecho, con el proyecto de vida, con el género, en fin, con todos y cada uno de los elementos que constituyen su identidad y su vida en sociedad. Cada persona tiene un concepto de sexualidad diferente que se ve influenciado por la sociedad, las culturas, la época, la historia y las tendencias individuales.

En este sentido diversos autores, entre los que destacan Rubio Aurioles (2014) y Murillo Gamboa (2017), coinciden en señalar que uno de los aspectos fundamentales o pilares de la sexualidad es la afectividad o vinculación afectiva. La cual ha sido poco considerada en los programas de Educación en Sexualidad, que se quedan solo en la genitalidad y le indexan la reproducción. Con ello solo impiden el pleno disfrute de este aspecto de la personalidad del individuo que podría darle significado y relevancia a su existencia y le podría garantizar el camino hacia su felicidad y realización.

Para Alonso (2005), la afectividad es un aspecto de la adaptación del individuo con funciones propias que orienta y matiza las acciones del sujeto; abarca todos los estados anímicos y todas las reacciones que se enraízan en el instinto y en el inconsciente, El componente afectivo es el eje medular de toda relación humana y del desarrollo de su sexualidad y, por ende, la forma en cómo se expresen las emociones. La claridad que se tenga de su importancia determinará la clara conciencia y manejo adecuado de los sentimientos. El mundo de los afectos es 
como la bisagra de toda la personalidad. Es donde se une lo sensible con lo espiritual y apunta al equilibrio personal.

El objetivo de los afectos entre dos personas que se sienten atraídas entre sí es el amor y la entrega al otro. Durante mucho tiempo se creyó que lo decisivo era sólo la voluntad de querer hacerlo y el intelecto que racionalizaba el actuar. Es cierto que estos, en el orden objetivo de las cosas, son determinantes y están llamados a orientar e iluminar en definitiva el actuar. Pero ellos solos, de por sí, es poco lo que pueden hacer sin la integración de la vida afectiva y espiritual.

Es difícil para las personas señalar el momento exacto en el que percibieron, de manera consciente, por primera vez sensaciones placenteras asociadas a la vivencia de su sexualidad en el plano físico de su corporalidad. Esto suele darse de forma gradual, y sin la mediación de la voluntad con la intencionalidad para hacerlo. A veces, el origen de esas sensaciones ni siquiera está motivado por una atracción específica hacia otra persona. Existen muchos estímulos en el entorno que a través de los sentidos pueden percibirse y despertar esas sensaciones.

Cuando la conciencia comienza a tomar las riendas del accionar del individuo, las personas sienten, simplemente, que les pasa algo nuevo, que no saben muy bien cómo calificarlo. Si es una sensación física, una emoción, un sentimiento, etc. A veces puede costar relacionar estos sentimientos y sensaciones, que se perciben con la sexualidad propiamente dicha y solo se asocia con la visión sesgada que se tiene orientada hacia el placer carnal producto de lo que se ha aprendido en el hogar y mediatizado por elementos culturales y el continuo bombardeo de los medios de comunicación.

El experimentar estas sensaciones placenteras asociadas a la sexualidad puede tener su origen en estímulos del propio cuerpo del individuo al activarse su sensorialidad o puede ser producto de estímulos sensoriales provenientes de otra persona que, al comenzar a experimentarse, pueden generar confusiones. Esto sucede porque desde su infancia, los adultos significantes no lo han preparado para vivir su afectividad. Murillo Gamboa (2007) señala que desde el nacimiento los sexos son separados por condicionamientos que refuerzan los estereotipos de género impuestos por la sociedad. Luego en determinada edad se espera que ocurra el encuentro con el otro en circunstancias en las que ellos no han transitado por el encuentro intimo consigo mismo, para poder conocerse y ponderar las potencialidades de su ser biológico. El primer requisito es que para querer al otro hay que quererse a sí mismo.

Es evidente que la Educación en Sexualidad no aborda las dimensiones afectivas en la formación del ser del individuo; generalmente se enfatiza en lo cognitivo, Igual puede decirse del ámbito espiritual. De allí que es necesaria la construcción de una propuesta sólida y coherente, basada en la realidad que vive el país que se oriente hacia estos aspectos de la personalidad que han sido dejado de lado en la formación del individuo desde su hogar y la escuela. De esta forma es urgente establecer una vía que conduzca al establecimiento de relaciones afectivas significativas y edificantes que le garanticen a la persona bienestar consigo mismo y lo preparen para encuentros estables y duraderos con el otro. Esto exige conocer los ámbitos epistemológicos, axiológicos, ontológicos y metodológicos del abordaje de la 
Educación Integral de la Sexualidad centrada en el individuo, en su afectividad y espiritualidad.

\section{REFERENCIAS}

Alonso, M. T. (2005). Pedagogía para la primera infancia 3: la afectividad en el niño: manual de actividades preescolares. España: Editorial MAD.

Fundación Escuela de Gerencia Social (2006). Embarazo en Adolescentes. Venezuela. Disponible en http://cort.as/-L9qa

Laak Jan Ter (1996). Las cinco grandes dimensiones de la personalidad. En Revista de Psicología de la PUCP, XIV(2).

Ministerio del Poder Popular para la Educación (2010). Líneas Estratégicas Curriculares Para La Educación De La Sexualidad En El Subsistema De Educación Básica. Caracas, Venezuela: Editorial Ignaka.

Murillo Gamboa, M. (2008). Cómo enseñar sexualidad: Para aprender el lenguaje de la sexualidad y enseñarlo. México: Editorial Pax Mexico.

Organización de Estados Iberoamericanos (2012). Metas Educativas 2021: la educación que queremos para la generación de los bicentenarios. Disponible en: https://www.oei.es/Educacion/metas2021/documento-final

OEI (2016). Miradas sobre la Educación en Iberoamérica 2016. Avances en las Metas Educativas 2021. Disponible en:

https://www.oei.es/Educacion/Noticia/miradas-sobre-la-educacion-eniberoamerica-2016

Organismo Regional Andino De Salud - Convenio Hipólito Unanue (2008). Plan Andino De Prevención Del Embarazo En La Adolescencia (PLANEA). Disponible en: http://www.planandino.org/portal/?q=node/61

OMS (1983). Salud sexual. Disponible en:

https://www.who.int/topics/sexual_health/es/

Rubio Aurioles, E. (2014). Lo que todo clínico debe saber de Sexología. México: Edición y Farmacia.

UNFPA (2011). Prevención del embarazo adolescente, una mirada completa. 